The Astrophysical Journal, 638:1070-1079, 2006 February 20

(C) 2006. The American Astronomical Society. All rights reserved. Printed in U.S.A.

\title{
THE FORMATION AND EVOLUTION OF PLANETARY SYSTEMS (FEPS): DISCOVERY OF AN UNUSUAL DEBRIS SYSTEM ASSOCIATED WITH HD 12039
}

\author{
Dean C. Hines, ${ }^{1}$ Dana E. Backman, ${ }^{2}$ Jeroen Boumman, ${ }^{3}$ Lynne A. Hillenbrand, ${ }^{4}$ John M. Carpenter, ${ }^{4}$ Michael R. Meyer, ${ }^{5}$ \\ Jinyoung Serena Kim, ${ }^{5}$ Murray D. Silverstone, ${ }^{5}$ Jens Rodmann, ${ }^{3}$ Sebastian Wolf, ${ }^{3}$ Eric E. Mamajek, ${ }^{6}$ \\ Timothy Y. Brooke, ${ }^{4}$ Deborah L. Padgett, ${ }^{7}$ Thomas Henning, ${ }^{3}$ Amaya Moro-Martín, ${ }^{8}$ E. Stobie, ${ }^{5}$ \\ Karl D. Gordon, ${ }^{5}$ J. E. Morrison, ${ }^{5}$ J. Muzerolle, ${ }^{5}$ and K. Y. L. Su ${ }^{5}$ \\ Received 2005 March 3; accepted 2005 October 8
}

\begin{abstract}
We report the discovery of a debris system associated with the $\sim 30$ Myr old G3/5V star HD 12039 using Spitzer Space Telescope observations from 3.6-160 $\mu \mathrm{m}$. An observed infrared excess $\left(L_{\mathrm{IR}} / L_{*}=1 \times 10^{-4}\right)$ above the expected photosphere for $\lambda \gtrsim 14 \mu \mathrm{m}$ is fit by thermally emitting material with a color temperature of $T \sim 110 \mathrm{~K}$, warmer than the majority of debris disks identified to date around Sun-like stars. The object is not detected at $70 \mu \mathrm{m}$ with a $3 \sigma$ upper limit 6 times the expected photospheric flux. The spectrum of the infrared excess can be explained by warm, optically thin material comprised of blackbody-like grains of size $\gtrsim 7 \mu \mathrm{m}$ that reside in a belt orbiting the star at 4-6 AU. An alternate model dominated by smaller grains, near the blowout size $a \sim 0.5 \mu \mathrm{m}$, located at 30-40 AU is also possible but requires the dust to have been produced recently, since such small grains will be expelled from the system by radiation pressure in approximately a few times $10^{2} \mathrm{yr}$.
\end{abstract}

Subject headings: circumstellar matter — infrared: stars — planetary systems: protoplanetary disks stars: individual (HD 12039)

\section{INTRODUCTION}

Dust in the inner solar system can be seen via scattered light as the zodiacal glow visible to the naked eye near sunrise and sunset in dark clear skies. This dust is located within the terrestrial planet zone, arising primarily from collisions among rocky debris (asteroids) between Mars and Jupiter and secondarily from comet ejecta. Dust produced by collisions between remnant planetesimals is also expected to exist within the Kuiper Belt beyond Neptune. The region between 5 and $30 \mathrm{AU}$ in our solar system is kept relatively free of dust due to the dynamical action of the giant planets (Moro-Martín \& Malhotra 2002, 2003; Moro-Martín et al. 2005). Comparable planetary systems around other stars might be expected to produce similar dusty debris belts and gaps.

Examples of thermal emission from debris disks around fairly luminous main-sequence stars, including the prototype Vega (Aumann et al. 1984), were identified with IRAS (e.g., Walker \& Wolstencroft 1988; Backman \& Paresce 1993; Mannings \& Barlow 1998) and the Infrared Space Observatory (ISO; e.g., Habing et al. 2001; Spangler et al. 2001; Laureijs et al. 2002). Most of these systems are associated with A-type stars, since neither observatory had the sensitivity to detect the amount of material associated with Vega around low-luminosity Sun-like stars at distances beyond about 15-20 pc. Most debris systems

${ }^{1}$ Space Science Institute, 4750 Walnut Street, Suite 205, Boulder, CO 80301. 2 SOFIA, Mail Stop 211-3, NASA-Ames, Moffet Field, CA 94035-1000.

3 Max-Planck-Institut für Astronomie, D-69117 Heidelberg, Germany.

${ }^{4}$ Department of Astronomy, California Institute of Technology, Pasadena, CA 91125 .

5 Steward Observatory, The University of Arizona, 933 North Cherry Avenue, Tucson, AZ 85721.

${ }^{6}$ Harvard-Smithsonian Center for Astrophysics, 60 Garden Street, Cambridge, MA 02138

7 Spitzer Science Center, California Institute of Technology, Pasadena, CA 91125.

${ }^{8}$ Department of Astrophysical Sciences, Princeton University, Princeton, NJ 08540. identified to date are characterized by $\operatorname{cool}(T \sim 50-90 \mathrm{~K})$ disks, suggestive of material at gas giant or Kuiper Belt distances from their parent stars, $r_{\text {dust }} \sim 10-100$ AU (see, e.g., Backman \& Paresce 1993; Decin et al. 2000, 2003; Lagrange et al. 2000; Zuckerman 2001, and references therein).

Since much of the zodiacal dust in our solar system arises from collisions in the asteroid belt (e.g., Grogan et al. 2001; Nesvorný et al. 2002, 2003), we might expect to observe excess emission from debris in a similar zone around other stars (Stern 1994; Zhang \& Sigurdsson 2003). However, only a handful of debris disks associated with less luminous, Sun-like stars have been found to exhibit peak infrared excess emission from warm dust $(T \sim 100-300 \mathrm{~K})$ suggestive of terrestrial zone material $\left(r_{\text {dust }} \sim 2-5 \mathrm{AU}\right)$.

Herein, we report the discovery of another example, which is associated with HD 12039 (DK Cet, HIP 9141), a young ( $\approx 30 \mathrm{Myr}$ ) G3/5V star (Houk \& Smith-Moore 1988) at $42 \pm$ 2 pc (Perryman 1997). The infrared excess was identified in the initial enhanced data product release from our Spitzer Space Telescope (Spitzer; Werner et al. 2004) legacy science program titled FEPS (Formation and Evolution of Planetary Systems; Meyer et al. 2004; M. R. Meyer et al. 2006, in preparation; Hines et al. 2004b).

Sections 2 and 3 briefly describe the FEPS program and the Spitzer observations of HD 12039. Section 4 describes our discovery of the debris system associated with HD 12039 and presents some of the properties of the star and its infrared excess. We further characterize and model the debris system in $\S 5$. Section 6 explores the origin of the emitting material and how HD 12039 fits into models of disk evolution and planet formation. We also compare HD 12039 to other warm debris systems associated with Sun-like stars.

\section{THE FEPS PROGRAM AND V1.1 SAMPLE}

The FEPS Spitzer Legacy program encompasses observations of 328 Sun-like $\left(\sim 0.8-1.5 M_{\odot}\right)$ stars with ages ranging 
from $\sim 3$ Myr to 3 Gyr. The survey enables us to examine the frequency of stars that have circumstellar gas and dust, as well as to infer some of the properties of detected dust (Meyer et al. 2004; M. R. Meyer et al. 2006, in preparation).

Our ultimate goal is to assess whether debris systems such as that found in our solar system are common or rare around Sun-like stars in the disk of the Milky Way. FEPS uses all three Spitzer science instruments to provide spectral coverage from 3.6 to $70 \mu \mathrm{m}$ and includes $\lambda \sim 7-35 \mu \mathrm{m}$ low-resolution Infrared Spectrograph (IRS; Houck et al. 2004) spectra. We also obtain $160 \mu \mathrm{m}$ observations for a subset of the FEPS stars located in areas of low-infrared cirrus background. In addition, FEPS provides models of the photospheric emission that have been fit to available optical and near-infrared photometry for each star.

The initial FEPS data release contains $\approx 10 \%$ (33 stars) of our total sample. A detailed description of the observing strategy, data analysis, and best-fit, Kurucz photospheric emission models for these stars is provided in the FEPS Explanatory Supplement (ver. 1.1; Hines et al. 2004b). ${ }^{9}$ The Spitzer data and documentation for the FEPS program are available directly from the Spitzer Legacy Science Archive. ${ }^{10}$

The 33 stars were randomly chosen by the Spitzer Science Center (SSC) for scheduling between UT 2004 May and UT 2004 September. They cover a wide range of properties within the selection criteria used for the entire FEPS program and therefore provide a good subsample from which to begin looking for interesting objects.

In particular, these stars cover a broad range of effective temperatures, $5121 \mathrm{~K} \leq T_{33} \leq 6227 \mathrm{~K}$ (compared with $4299 \mathrm{~K} \leq$ $T_{\text {FEPS }} \leq 6769 \mathrm{~K}$ for the entire sample), have an age range $7.2 \lesssim$ $\log ($ age $) \lesssim 9.2(\mathrm{yr})$, and lie at distances $20 \leq d \leq 127$ pc. FEPS stars with younger ages, $6.2 \leq \log$ (age) $\lesssim 7.2$, and greater distances, $d \gtrsim 130 \mathrm{pc}$, are primarily cluster members and are less well represented by these 33 stars.

FEPS results to date include: (1) the frequency of young, optically thick disks detected with the Infrared Array Camera (IRAC; Silverstone et al. 2006); (2) cool debris systems having excesses detected only at Multi-band Imaging Photometer (MIPS; Rieke et al. 2004) $70 \mu \mathrm{m}$ and therefore comparable to our own Kuiper Belt (Meyer et al. 2004; Kim et al. 2005); and (3) constraints on the amount of remnant gas in the disk surrounding HD 105 (Hollenbach et al. 2005). We have also investigated the occurrence of debris disks in the 100 Myr old Pleiades star cluster (Stauffer et al. 2005). We focus the current study on the Sun-like star HD 12039, which exhibits dust emission with temperatures that are warmer than those found in typical disks around other stars.

\section{SPITZER OBSERVATIONS OF HD 12039}

In this section we briefly review the observing strategy and data reduction methods specifically for HD 12039. Observations of the other 32 stars were executed and processed in a similar manner. Additional details are presented in the Explanatory Supplements for the FEPS V1.1 and V2.0 data releases (Hines et al. 2004b, 2005), and in Kim et al. (2005), J. Bouwman et al. 2006, in preparation, and Silverstone et al (2005) for MIPS, IRS, and IRAC, respectively.

\footnotetext{
9 Subsequent to the release of ver. 1.1, the IRS spectra were reprocessed by an updated SSC pipeline that significantly improved the calibration. The MIPS Instrument Team also released an improved data analysis pipeline that reduced the noise at $70 \mu \mathrm{m}$. These updated IRS and MIPS data are used herein and have been released publicly in FEPS ver. 2.0.

${ }^{10}$ At http://data.spitzer.caltech.edu/popular/feps.
}

MIPS observations of HD 12039 were obtained using the small-field photometry mode on UT 2004 July 11 at 24, 70, and $160 \mu \mathrm{m}$. Two cycles of $3 \mathrm{~s}$ data collection events (DCEs) at $24 \mu \mathrm{m}$, two cycles of $10 \mathrm{~s}$ DCEs at $70 \mu \mathrm{m}$, and four cycles of $10 \mathrm{~s}$ DCEs at $160 \mu \mathrm{m}$ were obtained.

After initial processing by the SSC S10.5.0 pipeline to provide reconstructed pointing information, the MIPS data were further processed using the MIPS Data Analysis Tool (DAT, ver. 2.9; Gordon et al. 2004, 2005). This includes the "enhancer" routines that correct for distortion in individual images and combine the images onto a subsampled mosaic. Aperture photometry using IDP3 (ver. 2.9; Schneider \& Stobie 2002) was performed on the individual $24 \mu \mathrm{m}$ DCE images and on the mosaic images at 70 and $160 \mu \mathrm{m}$. We adopt $14.7,29.7$, and $48 . \prime 0$ target aperture radii at 24,70 , and $160 \mu \mathrm{m}$, respectively. Background annuli from 29 ".4-41".7, 39".6-79".2, and 48."0-80".0 were used for the three bands, respectively. The median background per pixel was scaled to the appropriate target aperture size and subtracted from the summed target aperture flux for each star. The final flux in instrumental units was then corrected to an infinite aperture (MIPS Data Handbook, ver. 3.1; hereafter MDH3.1).

Random uncertainties in the background-subtracted estimates of on-source flux were estimated from the standard deviation of the mean of the multiple measurements for the $24 \mu \mathrm{m}$ observations. These "internal" uncertainties represent the precision of our measurements independent of the absolute flux calibration of Spitzer.

The "internal" uncertainties for the 70 and $160 \mu \mathrm{m}$ observations of HD 12039 were estimated by the rms pixel-to-pixel dispersion inside the background annulus measured on the mosaic image and scaled to the area of the target aperture.

Conversion to physical flux density units was performed with a simple multiplication of the instrumental total flux by the MIPS calibration factor as published currently in the MDH3.1. The MIPS absolute calibration uncertainties for the 24, 70, and $160 \mu \mathrm{m}$ wavelength bands are currently listed as $10 \%, 20 \%$, and $20 \%$, respectively, in the Spitzer Observer's Manual Version 4.6 (hereafter SOM V4.6), and in the MDH3.1. ${ }^{11}$ However, the median of the ratio of the measured $24 \mu \mathrm{m}$ flux densities to Kurucz-model predicted $24 \mu \mathrm{m}$ flux densities for the ensemble of 33 FEPS stars is 0.98 with a standard deviation 0.05 (§ 4). Assuming that the stars do not have infrared excesses, this small dispersion indicates that the MIPS $24 \mu \mathrm{m}$ absolute calibration is actually accurate to $\sim 5 \%$. This uncertainty estimate is consistent with $24 \mu \mathrm{m}$ measurements of 69 F5-K5 (IV and V) stars observed by the MIPS GTO science team (Beichman et al. 2005a; Bryden et al. 2006). Excluding the sole warm debris system star HD 69830, Bryden et al. find that the mean MIPS $24 \mu \mathrm{m}$ to predicted $24 \mu \mathrm{m}$ flux density ratio for the remaining 68 stars is 0.99 with a dispersion of 0.06 , in excellent agreement with our results. We conclude that the photometric calibration of our MIPS $24 \mu \mathrm{m}$ photometry relative to the best-fit Kurucz models is $5 \%-6 \%$. Since we do not detect the photospheres at 70 and $160 \mu \mathrm{m}$, we cannot independently derive uncertainties relative to Kurucz models. Therefore, we adopt the SSC-published 20\% absolute uncertainty for both the MIPS 70 and $160 \mu \mathrm{m}$ photometry. Finally, we calculate a total uncertainty in the physical flux density for each photometric measurement by adding the "internal" and calibration uncertainties in quadrature.

Low-resolution $(R=70-120)$ spectra were obtained on UT 2004 July 13 with IRS. We used an IRS high-accuracy, blue

\footnotetext{
11 The MDH3.1 states that the absolute calibration at $24 \mu \mathrm{m}$ is "better than
} 
TABLE 1

Spitzer РнотометRу OF HD 12039

\begin{tabular}{|c|c|c|c|c|c|c|}
\hline Band & $\begin{array}{l}\text { Wavelength }^{\mathrm{a}} \\
(\mu \mathrm{m})\end{array}$ & $\begin{array}{l}\text { Flux Density } \\
\text { (mJy) }\end{array}$ & $\begin{array}{l}\sigma_{\text {int }} \\
(\mathrm{mJy})\end{array}$ & $\begin{array}{l}\sigma_{\text {tot }}^{\mathrm{b}} \\
(\mathrm{mJy})\end{array}$ & $\begin{array}{l}\text { Flux Density Excess }{ }^{\mathrm{c}} \\
(\mathrm{mJy})\end{array}$ & Instrument \\
\hline $3.6 \ldots \ldots \ldots \ldots \ldots .$. & 3.535 & 777.50 & 1.43 & 31.10 & 71.51 & IRAC \\
\hline $4.5 \ldots \ldots \ldots \ldots \ldots \ldots$ & 4.502 & 456.17 & 1.61 & 22.81 & 26.17 & IRAC \\
\hline 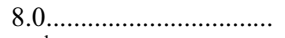 & 7.735 & 158.72 & 1.03 & 6.35 & -7.28 & IRAC \\
\hline $13^{\mathrm{d}} \ldots \ldots \ldots \ldots \ldots \ldots \ldots \ldots \ldots \ldots$ & 13.17 & 61.65 & 0.16 & 9.25 & 4.3 & IRS \\
\hline $24 \ldots \ldots \ldots$ & 23.68 & 24.65 & 0.22 & 1.50 & 6.1 & MIPS \\
\hline 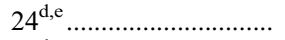 & 24.01 & 24.63 & 0.38 & 1.53 & 6.0 & IRS \\
\hline 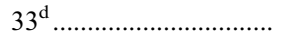 & 32.36 & 20.31 & 0.23 & 3.05 & 10.5 & IRS \\
\hline $70^{\mathrm{f}} \ldots \ldots \ldots \ldots \ldots \ldots$ & 71.42 & 4.5 & 2.5 & 2.7 & $\ldots$ & MIPS \\
\hline 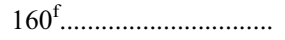 & 155.9 & -42 & 23 & 28 & $\ldots$ & MIPS \\
\hline
\end{tabular}

${ }^{\mathrm{a}}$ Weighted average wavelengths (SOM 4.6).

b The total uncertainty $\left(\sigma_{\mathrm{tot}}\right)$ is the rss of the "internal" measurement uncertainty (precision) and the "calibration" uncertainties (accuracy).

${ }^{\mathrm{c}}$ Calculated relative to the photospheric model described in the text.

${ }^{\mathrm{d}}$ Synthetic bandpass photometry with 1.6, 4.7, and $5 \mu \mathrm{m}$ band-widths (FWHM) for 13, 24, and $33 \mu \mathrm{m}$, respectively.

e The MIPS $24 \mu \mathrm{m}$ photometry has not had a color correction applied. The current value for the color correction would yield $F_{c}=1.056 \times F_{\text {obs }}=25.94 \mathrm{mJy}$, so the thermal excess above the photosphere would be $7.4 \mathrm{mJy}$.

${ }^{\mathrm{f}}$ Upper limits for the purposes of model fitting and plotting were calculated by $F($ upper $)=F(\mathrm{obs})+3 \sigma_{\text {tot }}$.

peak-up ( $1 \sigma$ uncertainty radius $\left.=00^{\prime \prime} 4\right)$ to acquire the star in the spectrograph slit, thus minimizing slit losses and assuring high photometric accuracy. Two nod positions per cycle were obtained in standard staring mode with one cycle for the short-low wavelength range (7.4-14.5 $\mu \mathrm{m})$, and three cycles for each of the two long-low wavelength ranges (14.0-21.3 and 19.5-38.0 $\mu \mathrm{m})$. The integration times were $6 \mathrm{~s}$ per exposure. The spectrum beyond $\sim 35 \mu \mathrm{m}$ suffers from high noise (Houck et al. 2004) and has been omitted.

The intermediate droopres products of the SSC pipeline S11.0.2 were processed within the SMART software package (Higdon et al. 2004). The background was subtracted using associated pairs of imaged spectra from the two nodded positions along the slit. This also subtracts stray light contamination from the peakup apertures and adjusts pixels with anomalous dark current relative to the reference dark frames. Pixels flagged by the SSC pipeline as "bad" were replaced with a value interpolated from an 8 pixel perimeter surrounding the bad pixel.

The spatially unresolved spectra were extracted using a 6.2 pixel fixed-width aperture in the spatial dimension for the first order of the short-wavelength low-resolution module, and 5.1 and 3.1 pixels for the first and second order of the long-wavelength low-resolution module, respectively. The spectra were calibrated using a spectral response function derived from IRS spectra and Kurucz stellar models for a set of 16 stars observed within the FEPS program that exhibit: (1) high signal-to-noise ratio $(\mathrm{S} / \mathrm{N})$ observations, (2) no residual instrumental artifacts, and (3) no signs of infrared excess. The absolute flux density scale was tied to calibrator stars observed by the IRS instrument team, but reduced as for the rest of the FEPS sample and referenced to calibrated stellar models provided by the SSC (see also Hines et al. 2004b, 2005; J. Bouwman et al. 2006, in preparation). Using this internally consistent approach enables us to improve the absolute flux calibration and reduces the noise on the adopted relative spectral response functions. This ensures that the uncertainties in the final calibration are dominated by photon noise and not by the uncertainties in the calibration. We estimate that the relative flux calibration across the spectrum is $\sim 1 \%-2 \%$. As for MIPS, a direct comparison of the IRS spectra for the 33 stars to the predicted flux densities from our best-fit Kurucz models indicates absolute uncertainties of $\approx 6 \%$ at $24 \mu \mathrm{m}(\S 3)$.

IRAC (Fazio et al. 2004) observations in channels 1, 2, and 4 (3.6, 4.5, and $8.0 \mu \mathrm{m})$ were obtained on UT 2004 July 19 using the $32 \times 32$ pixel subarray mode and a four-point random dither pattern, with an effective integration time of $0.01 \mathrm{~s}$ per image (frame time $=0.02 \mathrm{~s}$ ). The 64 images obtained at each of the four dither positions provided a total of 256 images of each star for a total integration time of $2.56 \mathrm{~s}$ in each channel.

All photometry was performed on the Basic Calibrated Data products from the SSC S10.5.0 data pipeline as described in the SOM4.6 and the Pipeline Description Document available through the SSC. Aperture photometry was performed using IDP3 with a 3."6 radius aperture centered on the target, and the background was estimated by the median of the pixels in a 12.2 $39^{\prime \prime}$ radius annulus centered on the source in each subarray image. This annulus circumscribes the $32 \times 32$ array and thus uses all pixels outside of a 10 pixel radius from the target. The background flux was normalized to the area of the target aperture and subtracted from the summed target aperture flux. The reported source flux is the mean of the 256 measures, corrected from a 3."6 radius to the calibration aperture using values published in Table 5.7 of the Infrared Array Camera Data Handbook Version 1.0. The "internal" uncertainties are estimated from the standard deviation of the 256 measurements and are typically $\lesssim 1 \%$. The ratios of measured to predicted flux densities for the 33 stars in the three IRAC bands are $1.08 \pm 0.04,1.04 \pm 0.05$, and $0.94 \pm 0.04$ for IRAC 3.6, 4.5, and $8.0 \mu \mathrm{m}$, respectively. This suggests that there are residual systematic offsets in the absolute calibration of the IRAC subarray mode. These are probably caused by gradients in the filter bandpasses across the detector (Quijada et al. 2004; Reach et al. 2005), which have yet been fully characterized for the subarray mode. Given these systematic offsets, we conservatively adopt the $10 \%$ absolute uncertainties as listed for the three bands in SOM 4.6.

The IRAC and MIPS photometry for HD 12039 is presented in Table 1 . We also present photometry centered at 13,24, and $33 \mu \mathrm{m}$ constructed from the IRS spectrum using rectangular bandpasses of 1.6, 4.7, and $5 \mu \mathrm{m}$ FWHM, respectively. These IRS flux densities are the error-weighted means with associated errors in the mean as appropriate.

\section{THE INFRARED EXCESS ASSOCIATED WITH HD 12039}

Figure 1 shows a plot of the $F_{\nu}(24 \mu \mathrm{m}) / F_{\nu}(K s)$ versus $F_{\nu}(4.5 \mu \mathrm{m}) / F_{\nu}(K s)$ flux density ratios for the 33 stars in the sample. Data are shown from both the MIPS $24 \mu \mathrm{m}$ band and the 


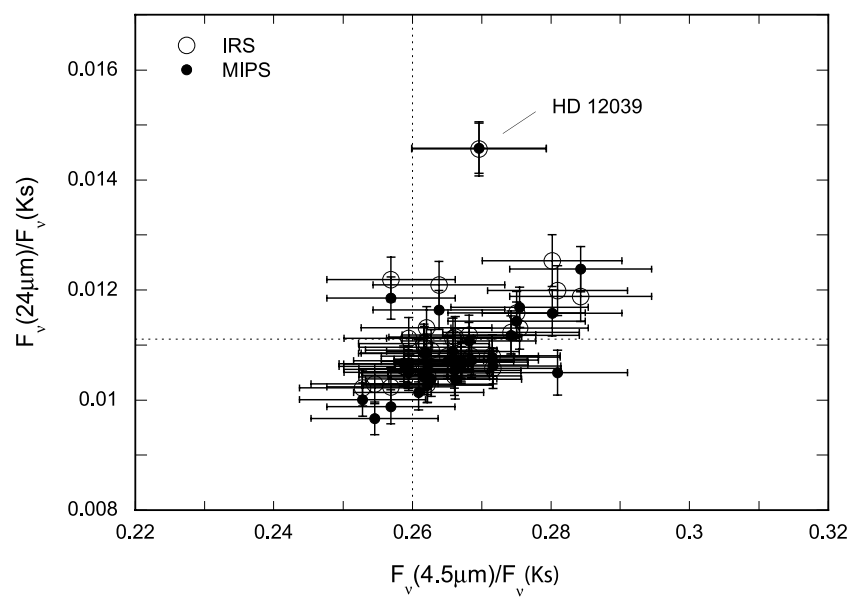

FIG. 1.-Flux ratio diagram $\left[F_{\nu}(24 \mu \mathrm{m}) / F_{\nu}(K s)\right.$ vs. $\left.\mathrm{F}_{\nu}(4.5 \mu \mathrm{m}) / F_{\nu}(K s)\right]$ for the 33 stars in the FEPS ver. 1.1 data release. Data are shown from both the MIPS $24 \mu \mathrm{m}$ broadband photometry and from a synthetic broadband point centered at $24 \mu \mathrm{m}$ formed from the IRS spectra. Note that the $F_{\nu}(4.5 \mu \mathrm{m}) / F_{\nu}(K s)$ ratio for HD 12039 is near the median for the other 32 stars consistent with photospheric colors, but the $F_{\nu}(24 \mu \mathrm{m}) / F_{\nu}(K s)$ ratio is anomalously high indicating a significant $24 \mu \mathrm{m}$ excess above the photosphere. Error bars represent $1 \sigma$ in the ratios calculated from the internal IRS and MIPS measurement uncertainties, and the total uncertainties of the 2MASS Ks flux densities. The $24 \mu \mathrm{m}$ excess from HD 12039 is detected in both MIPS and IRS independently and at different epochs. The dotted lines indicate the ratios predicted by the bestfit Kurucz model atmosphere for HD 12039. Stars with photospheric temperatures different than HD 12039 will have slightly different $F_{\nu}(4.5 \mu \mathrm{m}) / F_{\nu}(K s)$ ratios, which explains the dispersion in this quantity for the 33 stars in the sample.

synthetic IRS $24 \mu \mathrm{m}$ band. To construct the diagram, we used our most accurately calibrated IRAC band and the 2MASS $K s$-band, which is least affected by extinction. In addition, since only three of the stars lie outside of the dust-free Local Bubble $(d>80 \mathrm{pc})$, and all are within $150 \mathrm{pc}$, we do not expect extinction in the $K s$-band to cause us to identify spurious $24 \mu \mathrm{m}$ excesses. These ratios are independent of model atmosphere fits to the individual stars and will identify excess candidates regardless of any relative calibration offsets between the instruments.

Figure 2 shows the MIPS $F_{\nu}(24 \mu \mathrm{m}) / F_{\nu}(K s)$ ratio plotted versus the IRS $F_{\nu}(24 \mu \mathrm{m}) / F_{\nu}(K s)$ ratio, illustrating the independent instrumental calibration for IRS and MIPS as compared with

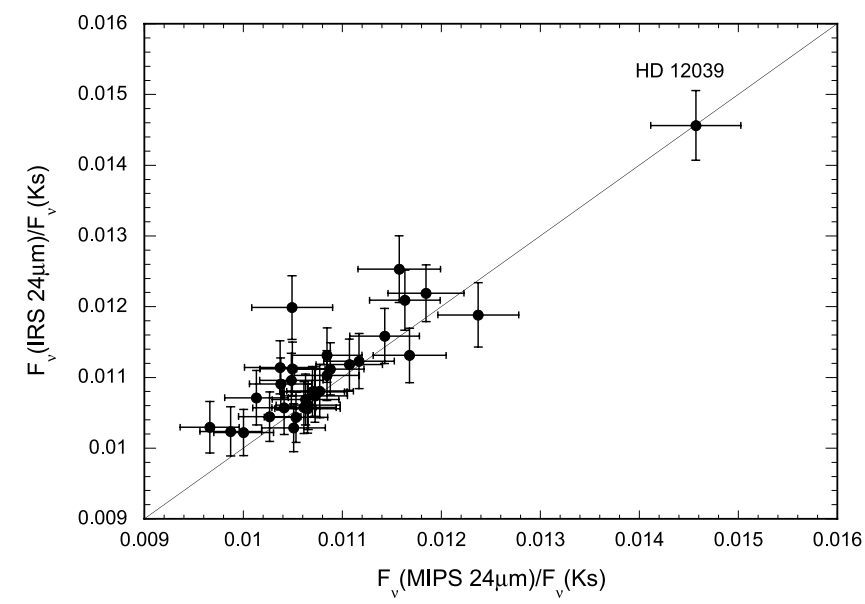

FIG. 2.-The $F_{\nu}(24 \mu \mathrm{m}) / F_{\nu}(K s)$ ratio for measurements from IRS and MIPS for the 33 stars in the FEPS ver. 1.1 data release. HD 12039 stands out clearly as having an anomalously high $F_{\nu}(24 \mu \mathrm{m}) / F_{\nu}(K s)$ ratio in both instruments indicating a significant $24 \mu \mathrm{m}$ excess above the photosphere. The solid line is of slope $=1$.
2MASS. The $30 \mathrm{Myr}$ old, G3/5 V star HD $12039^{12}$ is clearly distinguished in Figures 1 and 2, and apparently has significant excess emission at $24 \mu \mathrm{m}$ detected in both IRS and MIPS independently relative to the other 32 stars.

Since the MIPS and IRS data for each object were obtained $\approx 2$ days apart, the agreement between the flux density measurements for HD 12039 rules out a chance superposition of an asteroid in one of the observations as the explanation for the excess. Furthermore, inspection of 2MASS images shows that there are no other objects having $K s<15 \mathrm{mag}$ within $5^{\prime \prime}$ of the position of this star, and the $24 \mu \mathrm{m}$ image is indistinguishable from a single, isolated point source. Therefore, the measured $24 \mu \mathrm{m}$ emission is associated directly with HD 12039. The excess in the $F_{\nu}(24 \mu \mathrm{m}) / F_{\nu}(K s)$ ratios are observed at the 4.2 and $4.3 \sigma$ level relative to the median ratios for the other 32 stars from the MIPS $24 \mu \mathrm{m}$ and IRS $24 \mu \mathrm{m}$ photometry (Figs. 1 and 2). The probability of drawing this $\geq 4 \sigma$ result from a Gaussian distribution of data for these 33 targets is $<33 \times 1.3 \times 10^{-4}=4 \times 10^{-3}$.

As a further check on the reality of this excess at $24 \mu \mathrm{m}$, we compared the distributions of the ratios our measured MIPS and IRS $24 \mu \mathrm{m}$ flux densities to the flux densities predicted by Kurucz model atmospheres fit to optical and near-IR photometry for all 33 stars (Fig. 3). Excluding HD 12039, the median and

12 Details concerning our age determination for this star are given in the Appendix.

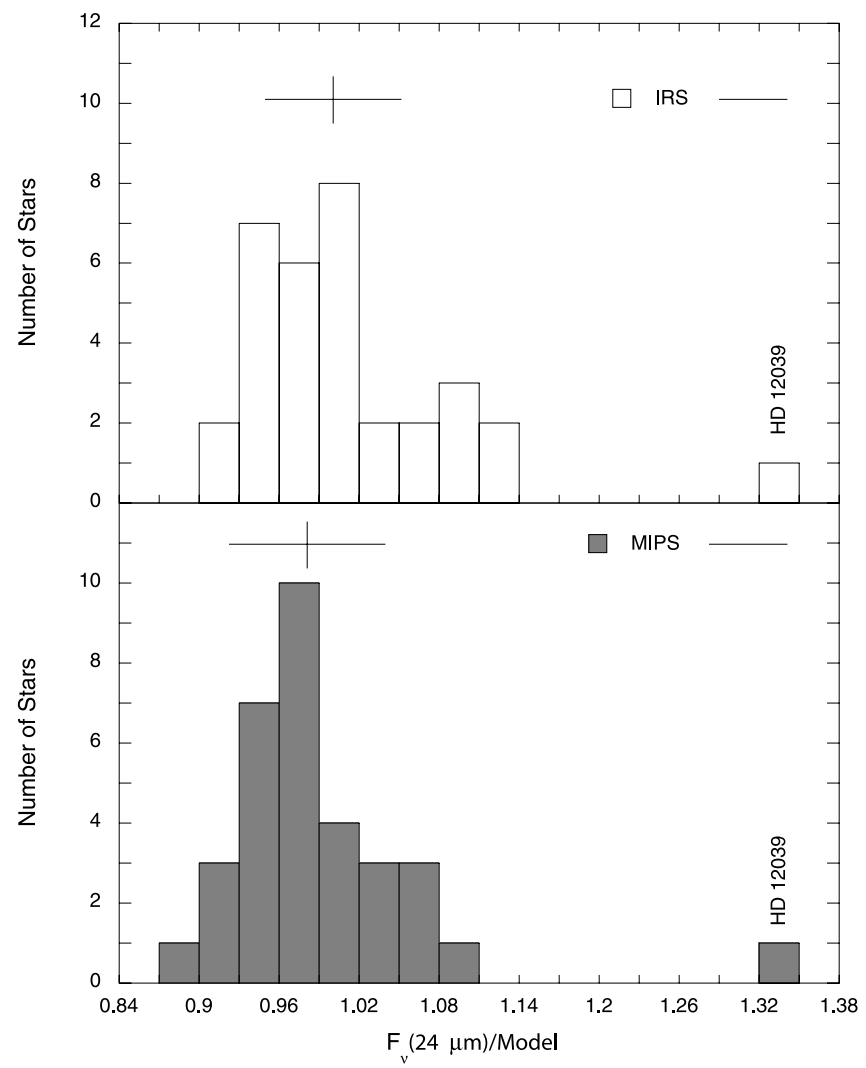

FIG. 3.- Histograms of the ratios of the measured IRS (top) and MIPS (bottom) $24 \mu \mathrm{m}$ flux densities to the predicted flux densities from our best-fit Kurucz models for the 33 stars in the FEPS ver. 1.1 data release. The bin width represents the average uncertainty in the ratios, and the crosses above the histograms indicate the median and standard deviation of the measurements excluding HD $12039(1.00 \pm 0.06$ and $0.98 \pm 0.05$ for IRS and MIPS, respectively). As in Figs. 1 and 2, HD 12039 stands out clearly as having a significant $24 \mu \mathrm{m}$ excess above the photosphere and is detected in both MIPS and IRS independently and at different epochs. 


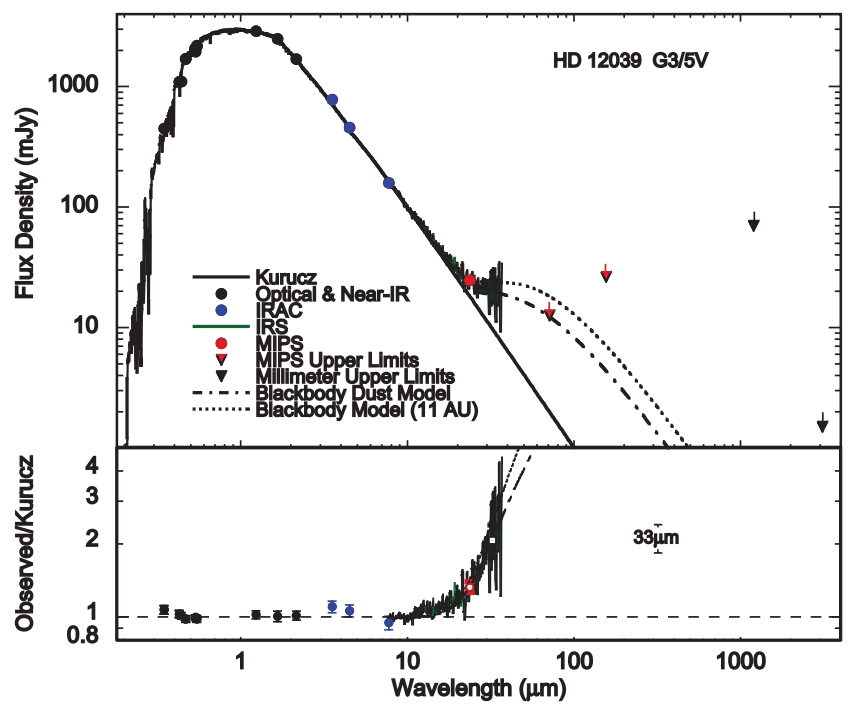

FIG. 4.-Spectrum of HD 12039. The top panel shows the ground and spacebased photometry (solid symbols), the IRS spectrum (green curve), and the Kurucz model that best fits the optical and near-IR photometry (solid black curve). The MIPS $24 \mu \mathrm{m}$ point has been color-corrected assuming a color temperature $T=110 \mathrm{~K}$ (see text). The $1 \sigma$ error bars for each point are plotted, but they are often smaller than the dot size in the top panel. We also show upper limits from MIPS 70 and $160 \mu \mathrm{m}$ photometry, as well as upper limits at millimeter wavelengths (Carpenter et al. 2005). The upper limits represent the actual on-source measured flux densities minus the background (which can be negative) plus 3 times the total uncertainty including the absolute calibration uncertainty. Also shown is the best-fit emission model for simple blackbody grains (dot-dashed line), and a blackbody model with the outer radius extending to $11 \mathrm{AU}$ (dotted line). Note that this latter model violates the $3 \sigma$ upper limit at $70 \mu \mathrm{m}$ and thus is ruled out by the observations. The bottom panel shows the spectrum of HD 12039 divided by the Kurucz model. The error bars for the IRS $33 \mu \mathrm{m}$ synthetic photometric point are duplicated to the right of the actual point for convenience. The IR emission can be seen departing from the photosphere for $\lambda \gtrsim 12-14 \mu \mathrm{m}$.

standard deviations $(1 \sigma)$ of these distributions are $0.98 \pm 0.05$ and $0.99 \pm 0.06$ for MIPS $24 \mu \mathrm{m}$ and IRS $24 \mu \mathrm{m}$, respectively, suggesting that the MIPS and IRS absolute flux calibrations are accurate to $5 \%-6 \%$ relative to the best-fit Kurucz models.

The ratio of measured-to-Kurucz model $24 \mu \mathrm{m}$ flux densities obtained with both instruments for HD 12309 is $1.33 \pm 0.04$, and $\left[F_{\nu, \text { obs }}(24 \mu \mathrm{m})-F_{\nu \text {, model }}(24 \mu \mathrm{m})\right] / \sigma$ is 5.4 and 5.3 for MIPS and IRS, respectively, where $\sigma$ is the root sum squares (rss) of the standard deviation of the distributions in Figure 3 and the total measurement uncertainty for HD 12039. Based upon the above analysis, we conclude that HD 12039 has excess emission above its stellar photosphere at $24 \mu \mathrm{m}$.

Figure 4 shows the IRAC and MIPS photometry plus the IRS low-resolution spectrum of HD 12039 compared to the model photosphere. The object was not detected at $70 \mu \mathrm{m}$ with a $3 \sigma$ upper limit that is 6 times the expected photospheric flux. Upper limits are also shown for $160 \mu \mathrm{m}, 1.2 \mathrm{~mm}$, and $3.1 \mathrm{~mm}$.

The photospheric emission component of the spectrum in Figure 4 was fit with Kurucz model atmospheres (including convective overshoot) to the optical and near-infrared photometry. Predicted magnitudes were computed as in Cohen et al. (2003 and references therein) using the combined system response of detector, filter, and atmosphere (for ground-based observations). The best-fit model was computed in a least-squares sense with the effective temperature and angular diameter of the star as free parameters, $[\mathrm{Fe} / \mathrm{H}]$ fixed to solar metallicity, surface gravity fixed to the value appropriate for the adopted stellar age and mass ( $\log g=4$ in this case), and visual extinction fixed to 0 mag within the dust-free Local Bubble $\left(d_{*} \approx 42 \mathrm{pc}\right)$. The derived bestfit stellar model has $T_{\text {eff }}=5688 \mathrm{~K}$, consistent with HD 12039's catalog G3/5 V spectral type.

In comparing Spitzer data to the model photosphere we have adopted the weighted average wavelengths that correspond to the monochromatic flux density within the filter bandpasses according to the SOM4.6. Also, for the purposes of plotting the data, a color correction $\left(F_{c}=1.056 \times F_{\text {obs }}\right)$ has been applied to the MIPS $24 \mu \mathrm{m}$ point, assuming a color temperature $T=110 \mathrm{~K}$ estimated from the slope of the IRS spectrum from $24 \mu \mathrm{m}$ to $33 \mu \mathrm{m}$ (see below). ${ }^{13}$

The IRAC and short-wavelength IRS data for HD 12039 are consistent with the best-fit Kurucz model. However, the IRS spectrum begins to depart from the photosphere between 12 and $14 \mu \mathrm{m}$, passes through the MIPS $24 \mu \mathrm{m}$ datum, and continues to increase until at least $33 \mu \mathrm{m}$. This is shown more clearly in the bottom panel of Figure 4, where we have plotted the ratio of the measured flux densities to the model-predicted flux densities. We also show the synthetic $33 \mu \mathrm{m}$ photometric point from the IRS spectrum, which lies $\gtrsim 3.5 \sigma$ above the Kurucz model.

The lack of significant excess continuum emission for wavelengths $\lesssim 14 \mu \mathrm{m}$ and the measured $24 \mu \mathrm{m}$ excess together imply a maximum color temperature of $T_{c} \lesssim 160 \mathrm{~K}$. The slope and uncertainty between the MIPS $24 \mu \mathrm{m}$ and IRS $33 \mu \mathrm{m}$ broadband photometric points correspond to color temperatures $T_{c}=109_{-37}^{+48} \mathrm{~K}$, $\sim 110 \mathrm{~K}$. The $70 \mu \mathrm{m}$ upper limit constrains the $33-70 \mu \mathrm{m}$ color temperature to $\geq 80 \mathrm{~K}$.

In the next section we further characterize the infrared excess and explore circumstellar disk models. We also consider constraints on the nature of the thermally emitting material imposed by timescales for evolution of dust in the system.

\section{ANALYSIS}

\subsection{General Characteristics of the Dusty Debris System}

To further characterize the infrared excess, we assume that the emission originates from large grains that absorb and emit radiation efficiently at all relevant wavelengths; that is, we treat the grains as blackbodies (e.g., Aumann et al. 1984; Walker \& Wolstencroft 1988; Backman \& Paresce 1993). Blackbody emission at wavelengths as long as $40 \mu \mathrm{m}$ requires grain radii larger than $a \sim 7 \mu \mathrm{m}$, and for such grains the dust physical temperature $T_{\text {dust }}$ is the same as the color temperature.

Given the fitted color temperature of $T \sim 110 \mathrm{~K}$ and assuming blackbody grains in thermal equilibrium with the stellar radiation field, we calculate a typical grain location of $6 \mathrm{AU}$ from the star. We also derive an upper limit to the grain radial distance by considering the $3 \sigma$ upper limit to the $70 \mu \mathrm{m}$ flux density (Figs. 3 and 4). Only blackbody grain models with $R_{\text {out }}<11 \mathrm{AU}$ do not violate this constraint. Thus, the observed infrared excess spectrum for HD 12039 is consistent with a fairly narrow range of dust temperatures and radial annuli. We note that although the IR emission from HD 12039 may be dominated by such relatively large grains, the characteristic emission temperature of $110 \mathrm{~K}$ is low enough that the lack of solid state emission features at, e.g., 10 and $18 \mu \mathrm{m}$ from the bending and stretching modes of standard astronomical silicates does not exclude the presence of smaller grains.

The observed excess at $24 \mu \mathrm{m}$ and limit at $\sim 14 \mu \mathrm{m}$ excludes the presence of material at $T \gtrsim 200 \mathrm{~K}$ with more than $\sim 0.1$ times the surface density inferred for the material near $110 \mathrm{~K}$,

\footnotetext{
13 MIPS color-corrections are tabulated in the MIPS Data Handbook ver. 3.0
} available from the SSC. 
assuming that any material at higher temperatures and smaller radii from the star would have a surface density $\propto r^{0}$ as for Poynting-Robertson (P-R) drag. Similarly, the $70 \mu \mathrm{m}$ limit excludes material at $T \sim 50 \mathrm{~K}$ with more than $\sim 0.5$ times the total grain cross-sectional area of material at $110 \mathrm{~K} .{ }^{14}$ The fractional infrared luminosity of the excess across the wavelength range 24-70 $\mu \mathrm{m}$ is $f=L_{\mathrm{IR}} / L_{*}=1 \times 10^{-4}$.

\subsection{Disk Models}

We further investigate the HD 12039 debris system using the dust disk models of Wolf \& Hillenbrand (2003), which take into account absorption and emission from dust grains based on their optical properties as opposed to the simple blackbody assumption. In particular, we use "astronomical silicates" (Draine \& Lee 1984; Laor \& Draine 1993; Weingartner \& Draine 2001). We assume a volume density profile $n(r) \propto r^{-1}$ that corresponds to a disk with constant mass surface density $\Sigma(r) \propto r^{0}$, consistent with but not requiring dynamic control by P-R drag $(\S 5.3)$.

As discussed above, the wavelength at which the dust reemission departs significantly from the stellar photosphere $(\lambda \sim$ $14 \mu \mathrm{m})$ yields an initial estimate of the inner radius of the disk $R_{\text {in }}$. This is also the location of the warmest material, but the dust temperature of $T \sim 110 \mathrm{~K}$ is too low to produce detectable silicate emission features (see, e.g., Fig. 10 in Wolf \& Hillenbrand [2003] and their discussion of the effects of inner holes, thus maximum temperatures, on the predicted spectra of debris disks).

To explain the spectrum of the infrared excess in HD 12039, we calculated the inner and outer disk radii and corresponding total dust mass for a suite of single-size-grain models with particle radii $a=0.1,0.5,1,3,7,25,50$, and $100 \mu \mathrm{m}$. We applied the Levenberg-Marquardt algorithm (Marquardt 1963; Markwardt 2003) to find the models that best fit the observations of HD 12039 in a $\chi^{2}$ sense (J. Rodmann et al. 2006, in preparation). The parameters of these models are presented in Table 2 and illustrated graphically in Figure 5. Models assuming small grains require large orbital radii from the $\operatorname{star}\left(R_{\text {in }} \approx 10-40 \mathrm{AU}, R_{\text {out }} \approx 20-50 \mathrm{AU}\right)$, because these small grains have low radiative efficiencies compared with blackbody grains. For grain sizes $a \geq 7 \mu \mathrm{m}$, we find values of $R_{\text {in }}=4 \mathrm{AU}$ and $R_{\text {out }}=6 \mathrm{AU}$, approximately independent of particle size, consistent with the simple blackbody dust model. Table 2 and Figure 5 also show that a model assuming $a=7 \mu \mathrm{m}$ grains ( located between 4-6 AU) yields the minimum dust mass. These single-size-grain models illustrate the inherent degeneracies in fitting spectral data alone without independent

\footnotetext{
14 In this case we can only place limits on dust emitting at a single temperature; there are no constraints on the surface density.
}

TABLE 2

Model Parameters for the Debris System HD 12039

\begin{tabular}{ccccc}
\hline \hline Model & $\begin{array}{c}\text { Grain Radius } \\
(\mu \mathrm{m})\end{array}$ & $\begin{array}{c}\text { Inner Disk Radius } \\
(\mathrm{AU})\end{array}$ & $\begin{array}{c}\text { Outer Disk Radius } \\
(\mathrm{AU})\end{array}$ & $\begin{array}{c}\text { Total Mass } \\
\left(10^{-4} M_{\text {earth }}\right)\end{array}$ \\
\hline $1 \mathrm{a} \ldots \ldots \ldots$. & 0.1 & 40 & 50 & 0.26 \\
$1 \mathrm{~b} \ldots \ldots \ldots$. & 0.5 & 27 & 40 & 0.1 \\
$1 \mathrm{c} \ldots \ldots \ldots$. & 1 & 20 & 31 & 0.08 \\
$1 \mathrm{~d} \ldots \ldots \ldots$. & 3 & 11 & 19 & 0.07 \\
$1 \mathrm{e} \ldots \ldots \ldots$. & 7 & 4.0 & 6.0 & 0.02 \\
$1 \mathrm{f} \ldots \ldots \ldots$. & 25 & 3.7 & 6.0 & 0.1 \\
$1 \mathrm{~g} \ldots \ldots \ldots .$. & 50 & 3.8 & 6.1 & 0.2 \\
$1 \mathrm{~h} \ldots \ldots \ldots .$. & 100 & 3.9 & 6.0 & 0.4 \\
$2 \ldots \ldots \ldots \ldots$. & $0.4-1000$ & 28 & 40 & 4.5 \\
\hline
\end{tabular}

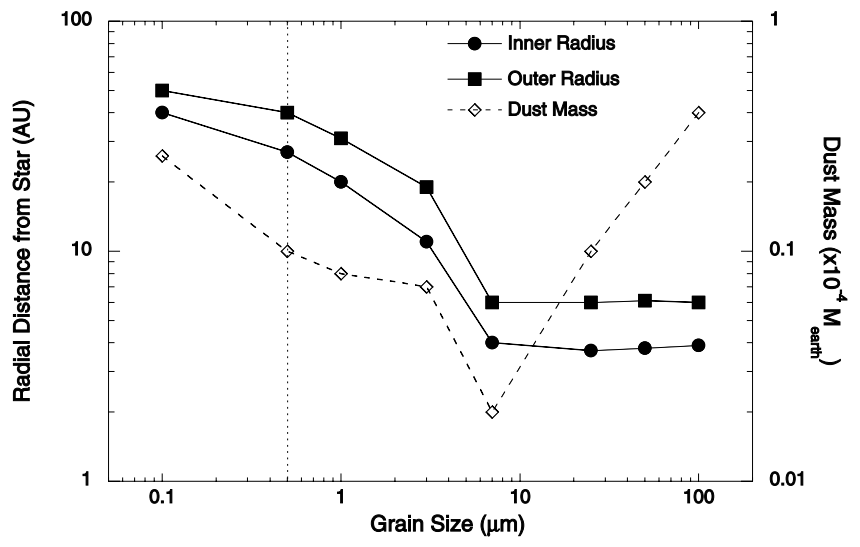

FIG. 5.- Single-size-grain debris disk models for HD 12039 following the prescription outlined in Wolf \& Hillenbrand (2003). The models are discussed in $\S 5.2$, and their parameters are listed in Table 2 . The inner and outer radial distances of the disk from HD 12039 and the associated dust mass for each disk model are plotted as a function of grain size. The vertical dotted line labels the blowout size $(a=0.5 \mu \mathrm{m})$ for silicate grains; smaller grains are expelled from the HD 12039 system by radiation pressure on timescales $\lesssim$ few hundred years. Note that grains with $a \geq 7 \mu \mathrm{m}$ behave as blackbodies and orbit in a belt from 4-6 AU. The model with $a=7 \mu \mathrm{m}$ grains represents the minimum dust mass.

morphological information such as direct imaging of the systems in thermal emission or scattered light.

Real systems in which the dust is produced by collisions of parent bodies will likely be composed of a distribution of particle sizes (Dohnanyi 1969; Williams \& Wetherill 1994; Tanaka et al. 1996). Therefore, we also modeled the infrared excess for HD 12039 using a power-law grain size distribution $n(a) \propto a^{-p}$ that included small grains of radius $a_{\min } \approx 0.4 \mu \mathrm{m}$ (just smaller than the blowout size; see below) and a maximum grain size of $a_{\max }=1000 \mu \mathrm{m}$ (just larger than can efficiently radiate at Spitzer wavelengths). The power-law exponent was set to $p=$ 3.5 , which is produced by a collisional cascade. For such a distribution, most of the opacity is supplied by the smallest grains and most of the mass in is contained in the largest grains. For these assumptions the best-fit inner and outer disk radii are $R_{\text {in }}=$ $28 \mathrm{AU}$ and $R_{\text {out }}=40 \mathrm{AU}$, respectively (Table 2). In general, more mass is required by this model than by the single-size-grain models considered above because of the inclusion of very large grains with small ratios of emitting area to mass.

Disk models and the photosphere-subtracted Spitzer data for HD 12039 are shown in Figure 6. All of the models require an inner hole radius $R_{\text {in }} \geq 4 \mathrm{AU}$, but we are unable to choose between the various models from the spectral fitting alone. We therefore consider, in the next section, physical scenarios for dust production and migration that lead us to a preferred model.

\subsection{Estimating Timescales for Grain Evolution}

We further constrain properties of the dust debris system around HD 12039 by considering the interaction between dust grains and radiation from the star (e.g., Burns et al. 1979; Backman \& Paresce 1993; Wyatt et al. 1999, and references therein; Thébault et al. 2003). Assuming there is no remnant gas in the system, typical astrophysical silicates with radius a $<0.5 \mu \mathrm{m}$ and density $\rho=2.5 \mathrm{~g} \mathrm{~cm}^{-3}$ produced by parent bodies in circular orbits at any distance surrounding a star with the luminosity and mass of HD 12039 will be expelled by radiation pressure. When grains travel a factor of 4 farther from the star than from where they originated, their equilibrium temperature 


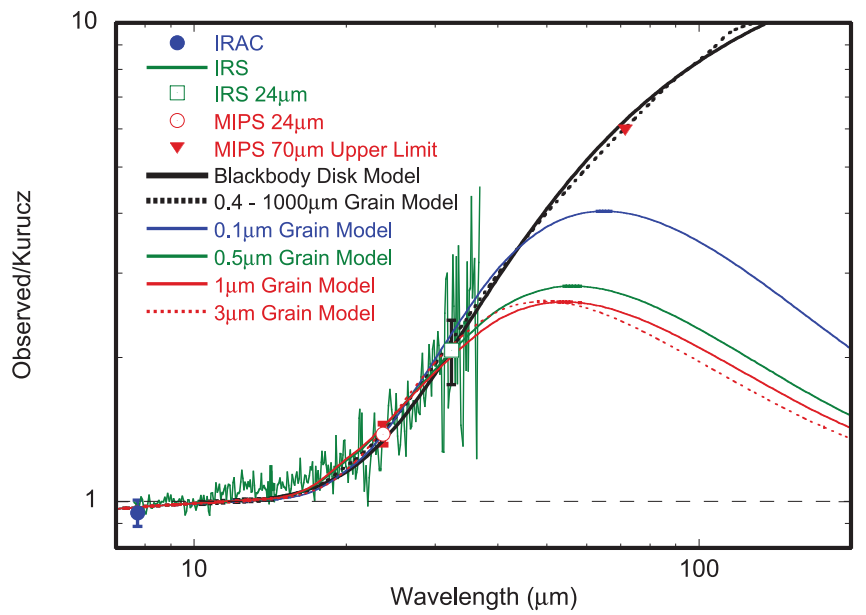

FIG. 6.-Spectrum of HD 12039 compared with spectra produced by the disk models from Table 2, all divided by the best-fit Kurucz model atmosphere. Several single-size-grain models are depicted as well as a model composed of grains with a power-law distribution of sizes from $0.4-1000 \mu \mathrm{m}$ (Table 2; see text). The best-fit blackbody model is also shown for comparison. The singlesize-grain dust models with particle sizes $a \geq 7 \mu \mathrm{m}$ from Table 2 are indistinguishable from the simple blackbody model and are not plotted independently in this figure.

falls by a factor of 2 and they are no longer important contributors to the observed infrared emission. The relevant timescales for this movement are $\sim 8$ and $\sim 80 \mathrm{yr}$ for grains starting at 6 and 30 AU, respectively.

Ice grains $\left(\rho \sim 1.0 \mathrm{~g} \mathrm{~cm}^{-3}\right)$, assumed to be "dirty" with albedos similar to silicates, will be similarly removed if $a<$ $1.5 \mu \mathrm{m}$. Therefore, the presence of grains smaller than the blowout size would signal a very recent collisional event.

In addition to radiation pressure expelling small grains from the system, P-R drag will cause inward migration of particles in the absence of remnant circumstellar gas. The timescales for silicate grains $\left(\rho=2.5 \mathrm{~g} \mathrm{~cm}^{-3}\right)$ to spiral into the star from a release radius $r_{\text {dust }} \sim 6 \mathrm{AU}$ are $t_{\mathrm{P}-\mathrm{R}} \sim 7 \times 10^{4}$ or $\sim 5 \times 10^{5} \mathrm{yr}$ for grains with $a \sim 1 \mu \mathrm{m}$ (a bit above the blowout size) or $a \sim 7 \mu \mathrm{m}$ (the minimum blackbody emitter size), respectively. This is much longer than the blowout time $\left(\lesssim 10^{2} \mathrm{yr}\right)$, but still short compared to the $t \sim 30 \mathrm{Myr}$ age of the system. Similar sized grains at 3040 AU would have P-R timescales $\sim 1-10 \mathrm{Myr}$, again shorter than the system age. In either distance regime, small particles are removed on sufficiently short timescales that the material must be replenished if we are witnessing a steady state. Alternatively, as mentioned above, the dust could have been produced by a relatively recent event.

The dust fractional surface density $\left(\mathrm{m}^{2}\right.$ cross section per $\mathrm{m}^{2}$ of disk) near the minimum orbital radius $R_{\min }$ is numerically roughly equal to the fractional luminosity in the excess $f=$ $L_{\mathrm{IR}} / L_{*}$ (Backman 2004), i.e., $f \sim 1 \times 10^{-4}$. From this we can explore the relative importance of P-R drag compared with grain evolution caused by mutual collisions (e.g., Wyatt et al. 1999; Wyatt \& Dent 2002; Wyatt 2005). Considering our two primary disk models discussed above, the dust mutual collision timescales in this system are approximately $2 \times 10^{4} \mathrm{yr}$ for $7 \mu \mathrm{m}$ grains at $r_{\text {dust }}=6 \mathrm{AU}$, and $\approx 2 \times 10^{5} \mathrm{yr}$ for $0.5 \mu \mathrm{m}$ grains at $r_{\text {dust }}=$ 30 AU with scaling as $r^{1.5}$ (Backman \& Paresce 1993). At $30 \mathrm{AU}$, the collision and P-R timescales are comparable, so material moves toward the star from its region of origin before collisions can modify it significantly. At 6 AU the collision timescale is 25 times smaller than the P-R timescale, so collisions can grind material down to the blowout size before the larger grains migrate toward the star via P-R drag. This creates a natural paucity of material inside the inner radius of the parent body orbits and could explain the observed lack of significant material at hotter temperatures (i.e., smaller orbital radii). This mechanism for keeping inner holes clear may operate in other well-studied disks (e.g., Wilner et al. 2002; Dominik \& Decin 2003; Wyatt 2005).

In this analysis we have not considered corpuscular drag from collisions with the stellar wind from HD 12039, which would increase the minimum grain blowout size. Even for young Sunlike stars, corpuscular drag forces are thought to be small compared with radiation forces $\left(F_{\text {wind }} / F_{\mathrm{P}-\mathrm{R}} \sim 0.35\right.$; Gustafson 1994$)$. Very tiny grains (e.g., $a<0.05 \mu \mathrm{m}$ for silicates) would be inefficient absorbers at the peak wavelengths of the stellar spectrum and perhaps stable against radiation blowout, but possibly vulnerable to ionization and resulting magnetic forces in ways that are poorly understood. We can assume that silicate grains with radii at least over the range $0.05<a<0.5 \mu \mathrm{m}$ (or dirty ice grains with $0.1<a<1.5 \mu \mathrm{m}$ ) are unstable against ejection from this system.

\section{DISCUSSION}

\subsection{The Debris System}

Our inferred properties of the debris associated with HD 12039 assume that dust dynamics are dominated by interactions with the stellar radiation field and are not determined by dustgas dynamics. This requires the gas-to-dust ratio to be $<0.1$ (e.g., Takeuchi \& Artymowicz 2001; Klahr \& Lin 2001). While this is consistent with the known properties of HD 12039 (no signatures of active accretion in the stellar spectrum and no strong mid-IR atomic or molecular emission-line features), detailed searches for remnant gas have not been made. With this caveat, the short grain evolution timescales relative to the system age imply that the dusty debris consists primarily of second-generation grains released by collisions and/or sublimation of larger parent bodies.

We have presented a range of possible models that successfully fit the thermal excess, but two are of particular interest because they represent end points of a plausible range of grain physical characteristics: (1) large "blackbody" grains $(a \geq 7 \mu \mathrm{m})$ located between 4 and $6 \mathrm{AU}$ from the star and (2) grains with sizes $0.4 \leq a \leq 1000 \mu \mathrm{m}$ characterized by a power-law distribution and located between 28 and 40 AU. Without resolved images of the debris system or more sensitive searches for mineralogical features, we cannot distinguish between these models with confidence. However, the surface area in model 2 with the wide belt and a grain size distribution that is dominated by grains near the blowout size would require either: $(a)$ a large mass ( $\left.>100 M_{\text {Earth }}\right)$ of parent bodies in collisional equilibrium to maintain the small-grain population against rapid blowout and P-R drift or $(b)$ the discovery of a large transient dust signature observable only for a few hundred years and due to recent collisional event.

Based on planetesimal accretion code models of a minimummass solar nebula (MMSN) surrounding a Sun-like star, Kenyon $\&$ Bromley $(2004,2005)$ have shown that the growth of planetesimals in the terrestrial zone can produce $\sim 1$ and $\sim 3$ mag infrared excesses at 10 and $24 \mu \mathrm{m}$, respectively. The peak emission occurs from $\sim 10^{4}$ to $\sim 10^{6} \mathrm{yr}$ when the largest planetesimals have reached $\sim 2000 \mathrm{~km}$ in size. By $10^{7} \mathrm{yr}$, the excess is nearly gone at $10 \mu \mathrm{m}$, but persists at $24 \mu \mathrm{m}$ until $\sim 10^{8}$ yr. Importantly, even though the overall dust production (thus IR emission) 
TABLE 3

Terrestrial Planetary Debris Systems

\begin{tabular}{|c|c|c|c|c|c|c|}
\hline Star & Spectral Type & $\begin{array}{l}\text { Age } \\
\text { (Myr) }\end{array}$ & $\begin{array}{l}T_{\text {dust }} \\
(\mathrm{K})\end{array}$ & $\begin{array}{c}r_{\text {dust }} \\
(\mathrm{AU})\end{array}$ & $\begin{array}{c}L_{\mathrm{IR}} / L_{*} \\
\left(\times 10^{-4}\right)\end{array}$ & Reference \\
\hline HR 4796A........................... & $\mathrm{A} 0 \mathrm{~V}$ & $8-10$ & 110 & 35 & 50 & 1 \\
\hline$\zeta$ Lep & $\mathrm{A} 2 \mathrm{~V}$ & 300 & 370 & $<6$ & 1.7 & 2 \\
\hline 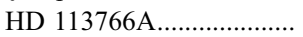 & F3V & 15 & 350 & $0.4-6$ & $150^{\mathrm{a}}$ & 3 \\
\hline 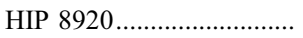 & G0V & 300 & 650 & $0.4-1$ & 400 & 4 \\
\hline 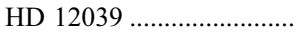 & $\mathrm{G} 3 / 5 \mathrm{~V}$ & 30 & 110 & $4-6$ & 1 & 5 \\
\hline HD 98800B....................... & $\mathrm{K} 7 \mathrm{~V}$ & $8-10$ & 160 & $2-4$ & 1900 & 6,7 \\
\hline 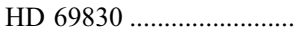 & $\mathrm{K} 0 \mathrm{~V}$ & 2000 & 207 & 1 & 3 & 8 \\
\hline Sun & G2V & 4500 & $150-170$ & $1-4$ & 0.001 & $9,10,11$ \\
\hline
\end{tabular}

${ }^{a}$ C. Chen 2005, private communication.

References.-(1) Jura et al. 1993; (2) Chen \& Jura 2001; (3) Chen et al. 2005; (4) Song et al. 2005; (5) this paper; (6) Low et al. 1999; (7) Koerner et al. 2000; (8) Beichman et al. 2005b; (9) Fixsen \& Dwek 2002; (10) Reach 1992; (11) Reach et al. 2003.

declines, individual collisions between $10-100 \mathrm{~km}$ bodies will produce short-lived $\sim 0.5-1.5$ mag signatures (see Fig. 3 in Kenyon \& Bromley 2004, 2005). The observed $\sim 0.3$ mag excess at $24 \mu \mathrm{m}$ in HD 12039 is roughly consistent with this scenario.

In contrast, it is harder to grow large bodies at farther distances from the star. Collisions at $30 \mathrm{AU}$ are predicted not to be massive enough in general to produce an observable excess above the photosphere at $24 \mu \mathrm{m}$ (Kenyon \& Bromley 2005). These considerations suggest that our model 1 is the most plausible explanation for the infrared excess in HD 12039.

The lack of material much warmer than $\sim 110 \mathrm{~K}$ in the HD 12039 system could be explained by several mechanisms. First, this temperature is approximately equal to the threshold for the onset of rapid sublimation of micron-sized water ice grains. Thus, if the dust parent bodies are primarily icy, the inner edge of the observed debris distribution could be determined by grain evaporation. Second, as discussed in $\S 5.3$, the timescale on which grains collide and produce fragments small enough for rapid blowout is an order of magnitude shorter than the P-R timescale at $6 \mathrm{AU}$, but the timescales are comparable at $30 \mathrm{AU}$. This suggests that the inner belt model could be self-limited. Dust grains would collide in situ before drifting inward a significant distance from their production location (Wyatt 2005), and the small grains would be blown out by radiation pressure. The existence of a planetesimal belt surrounding HD 12039 might require the presence of a nearby giant planet to stop the planet formation process, as is understood to be the case with Jupiter and the asteroid belt in our solar system. Third, a planet could exist at the inner edge of the debris annulus and consume/scatter inbound grains (e.g., Moro-Martín et al. 2005).

At the distance of HD 12039 , and with $L_{\mathrm{IR}} / L_{*} \approx 1 \times 10^{-4}$ in the range of objects detected previously in scattered light with HST (e.g., Schneider et al. 1999; Ardila et al. 2004; Fraquelli et al. 2004), the debris disk should be easily resolved in scattered light by coronagraphic imaging with NICMOS aboard HST, if it is composed of small grains at $30 \mathrm{AU}$. A null detection would strengthen the case that the material is concentrated in a belt at 4-6 AU. NICMOS (and ground-based adaptive optics) imaging should also place limits on the presence of a planet perturber that may be responsible for stirring up the debris.

\subsection{Terrestrial Planetary Debris Systems}

HD 12039 is the only object among the 33 Sun-like stars in this FEPS subsample that exhibits a prominent debris system at relatively warm temperatures approaching those of asteroidal ma- terial in our own solar system. It also apparently lacks significant material at cooler, Kuiper Belt-like temperatures. We know of only a handful of other such Terrestrial Planetary Debris Systems (Table 3).

Three systems are associated with stars more massive, and hence more luminous, than the Sun. The 8 Myr old A0 V star HR 4796A shows a strong infrared excess that is well modeled by a single-temperature blackbody $(T=110 \mathrm{~K})$ with grains orbiting at $\sim 35 \mathrm{AU}$ (Jura et al. 1998). Further high-resolution imaging of HR4796A in the infrared indicates the presence of a warmer $T=260 \pm 40 \mathrm{~K}$, compact dust component located at 4 AU (Wahhaj et al. 2005). The 10-20 Myr old F3 V star HD $113766 \mathrm{~A}$ is part of a wide (170 AU) binary that is a kinematic member of Lower Centaurus Crux association. The $T \sim$ $350 \mathrm{~K}$ material has been modeled by a debris belt $0.4-6 \mathrm{AU}$ from the star (Meyer et al. 2001; Chen et al. 2005). The $300 \mathrm{Myr}$ old A2 V star $\zeta$ Lep was first identified by Aumann \& Probst (1991) to possess an infrared excess, and Chen \& Jura (2001) find dust of several hundred kelvins, corresponding to a spatial distribution $<6-9$ AU.

Two additional warm debris systems are associated with stars less massive than the Sun: HD 98800 and HD 69830. The 8$10 \mathrm{Myr}$ old quadruple system HD 98800 in the TW Hya association is comprised of two spectroscopic binaries with $\mathrm{K}$ and M primaries (Torres et al. 1995; Soderblom et al. 1996, 1998). A circumbinary debris system first detected by IRAS (Walker \& Wolstencroft 1988; Zuckerman \& Becklin 1993) orbits HD 98800B (Koerner et al. 2000) is well characterized by a single $T \approx 160 \mathrm{~K}$ blackbody spectrum from $8 \mu \mathrm{m}$ to $7 \mathrm{~mm}$, implying debris orbiting at 2-4 AU (Low et al. 1999, 2005; Hines et al. 2004a). The presence of silicate emission features suggest that there is an additional population of small grains in this system (Skinner et al. 1992; Sitko et al. 2000; Schütz et al. 2005).

The 2 Gyr old K0 V star HD 69830 was also identified by IRAS as harboring a debris disk system (Mannings \& Barlow 1998). Similar to HD 12039, Spitzer observations of HD 69830 reveal a strong $24 \mu \mathrm{m}$ excess, and the $70 \mu \mathrm{m}$ flux density is consistent with the photosphere (Beichman et al. 2005b). In contrast to HD 12039, however, the IRS spectrum of HD 69830 has abundant mineralogical features similar to those found in ISO and ground-based mid-IR spectra of comet Hale-Bopp (Crovisier et al. 1996, 1997; Wooden et al. 1999a, 1999b). Beichman et al. (2005b) model the system with $a=0.25 \mu \mathrm{m}$ grains at about $3 \mathrm{AU}$ and suggest a recent dust-producing event.

Finally, the 30 Myr old G3/5 star HD 12039 and 300 Myr old G0 V star HIP 8920 (Song et al. 2005) each have masses and 
luminosities very similar to the Sun. HIP 8920 is extremely unusual, exhibiting a very hot $\left(T_{\text {dust }}=650 \mathrm{~K}\right)$ bright $\left(L_{\mathrm{IR}} / L_{*} \approx\right.$ 0.04) infrared excess. As for HD 69830, but unlike HD 12039, HIP 8920 shows a strong $10 \mu \mathrm{m}$ silicate emission feature, signaling the presence of grains smaller than $3 \mu \mathrm{m}$. Song et al. (2005) note that these small, hot grains would reside at $\sim 0.4$ AU.

Twenty isolated Sun-like stars in the $100 \mathrm{Myr}$ old Pleiades have been observed by the FEPS program to look for excess infrared emission (Stauffer et al. 2005). One "probable" and two "possible" detections at $24 \mu \mathrm{m}$ were found. These qualifiers result from the fact the Pleiades members are passing through a molecular cloud and are embedded in ISM dust that can be illuminated by the stars. Thus, they may have an associated infrared excess that is unrelated to the debris disk phenomenon. Also, the IRS 33 and $70 \mu \mathrm{m}$ upper limits suggest that these disks, if real, are fairly $\operatorname{cool} T \lesssim 85 \mathrm{~K}$ (Stauffer et al. 2005). Therefore, we have not explicitly included these stars in our list of warm debris disks.

The frequency of warm debris systems associated with Sunlike stars may be very coarsely estimated based on Spitzer results presented to date. So far, FEPS has found 1/33, Chen et al. (2005) find 1/40 (but possibly 3 total), and Beichman et al. (2005a, 2005b) and Bryden et al. (2006) have found 1/84. A MIPS GTO investigation of the $100 \mathrm{Myr}$ old open cluster M47 found $2 / 17$ Sun-like stars with a $24 \mu \mathrm{m}$ excess, one of which is sufficiently strong that it might be from terrestrial temperature dust (Gorlova et al. 2004). Another Spitzer investigation of 24 of the known stars within the TW Hydra Association (TWA; Low et al. 2005) confirmed that HR 4796A and HD 98800B are the only members of the association to harbor warm debris disks. ${ }^{15}$ As discussed above, FEPS observations of the Pleiades may have also uncovered $3 / 20$ objects with $24 \mu \mathrm{m}$ excess.

Compiling these results suggests that approximately $\sim 1 \%-$ $5 \%$ of Sun-like stars in the age range of $\sim 8-300$ Myr (plus HD 69830 at 2 Gyr) exhibit infrared excesses peaking at terrestrial temperatures. This rarity may reflect the short duty cycle of such events or, in steady state, an intrinsically low frequency of stars with the observed level of dust production in terrestrial planet-building zones. Kenyon \& Bromley (2005) find that the rate of collisions sufficiently large to produce a substantial $24 \mu \mathrm{m}$ signal is $\leq 10^{-5} \mathrm{yr}^{-1}$ for the period of terrestrial planet formation

\footnotetext{
15 A tentative $(2 \sigma)$ detection at 12 and $18 \mu$ m of a $T=170 \mathrm{~K}$ disk in TWA 17 by Weinberger et al. (2004) was not confirmed by MIPS $24 \mu \mathrm{m}$ observations that are consistent with the stellar photosphere (Low et al. 2005).
}

$t \sim 1-100$ Myr. $^{16}$ Therefore, the apparent paucity of Sun-like stars with bright thermal excesses and terrestrial temperatures may not be surprising.

\section{CONCLUSION}

We have discovered a debris system around the Sun-like star HD 12039 that exhibits some properties analogous to the dust associated with the asteroid belt in our solar system. While very small particles ( $a \lesssim 1 \mu \mathrm{m})$ at Kuiper Belt distances ( $r=30 \mathrm{AU})$ cannot be ruled out completely, we find that a debris belt consisting of $a \gtrsim 7 \mu \mathrm{m}$ grains orbiting HD 12039 at $r_{\text {dust }}=4-6 \mathrm{AU}$ provides the most plausible geometry. As for our solar system, such a narrowly confined zone of material may signal the presence of (as yet unseen) planets in the system.

HD 12039 joins only a handful of other debris disk systems with similar properties out of hundreds observed, suggesting that such systems are rare. If this rarity reflects the true incidence of terrestrial-zone debris, then perhaps asteroid belt analogs are uncommon. Alternatively, the collisional events that produce such strong infrared signals may be rare.

It is a pleasure to acknowledge all the members of the FEPS legacy science team for their contributions to the project. We thank C. Beichman for sharing information about HD 69830 prior to publication and an anonymous referee whose constructive criticism helped to improve our presentation. This work has used the SIMBAD databases, and is based in part on observations made with the Spitzer Space Telescope, which is operated by the Jet Propulsion Laboratory, California Institute of Technology, under NASA contract 1407 . Our investigation has also made use of data products from the Two Micron All Sky Survey (2MASS), which is a joint project of the University of Massachusetts and the Infrared Processing and Analysis Center, California Institute of Technology, funded by the National Aeronautics and Space Administration and the National Science Foundation. FEPS is pleased to acknowledge support from NASA contracts 1224768,1224634 , and 1224566 administered through JPL. The MPIA team is supported by the EU-RTNHPRN-CT-2002-00308 PLANETS network and the German Research Foundation (DFG) through the Emmy Noether grant WO 857/2-1.

\footnotetext{
${ }^{16}$ In our own solar system, the terrestrial planets are thought to have been $80 \%$ complete by an age of $30 \mathrm{Myr}$ (Kleine et al. 2002; Yin et al. 2002).
}

\section{APPENDIX}

\section{THE AGE OF HD 12039}

Song et al. (2003) claim that HD 12039 (DK Cet, HIP 9141) is a member of the 30 Myr old Tucana-Horologium association $\left(d_{*}=42 \pm 2\right.$ pc; Perryman \& ESA 1997). We independently confirmed the kinematic association by comparing the proper motion (Zacharias et al. 2004) and mean radial velocity ( $5.0 \pm 0.2 \mathrm{~km} \mathrm{~s}^{-1}$; Song et al. 2003; Nordstrom et al. 2004; White et al. 2006) to the values expected for a star at $d=42 \mathrm{pc}$ with the Tuc-Hor group space motion (Zuckerman \& Webb 2000).

An age of $\sim 30 \mathrm{Myr}$ is also consistent with other estimates. Position in the HR diagram $\left[T_{\text {eff }}=5688 \pm 60 \mathrm{~K}, \log \left(L / L_{\odot}\right)=\right.$ $-0.05 \pm 0.04 \mathrm{dex}$; Carpenter et al. 2005) compared to evolutionary tracks suggests an age of 28 Myr (D’Antona \& Mazzitelli 1997) to $33 \mathrm{Myr}$ (Baraffe et al. 1998); the mass predicted from either set of tracks is $1.02 M_{\odot}$.

Chromospheric activity and lithium depletion data corroborate the isochronal age estimate. The Ca II emission index has been measured to be $\log R_{\mathrm{HK}}^{\prime}=-4.21$ (D. R. Soderblom 2005, private communication) and $\log R_{\mathrm{HK}}^{\prime}=-4.14$ (White et al. 2006). This is broadly consistent with an age of $30 \mathrm{Myr}$, although the uncertainties in estimating ages from Ca II indices for ages $<300 \mathrm{Myr}$ are large.

The Li I $\lambda 6707$ equivalent width (Song et al. 2003; Wichmann et al. 2003; White et al. 2006) when interpolated among the distribution of Li I values for Sun-like cluster members (E. E. Mamajek 2006, in preparation) suggests an age of $15_{-10}^{+30}$ Myr. Considering all of these age indicators, we adopt an age of 30 Myr for HD 12039, based largely on its kinematic membership in the Tuc-Hor association. 
Ardila, D. R., et al. 2004, ApJ, 617, L147

Aumann, H. H., \& Probst, R. G. 1991, ApJ, 368, 264

Aumann, H. H., et al. 1984, ApJ, 278, L23

Backman, D. E. 2004, in ASP Conf. Ser. 324, Debris Disks and the Formation of Planets: A Symposium in Memory of Fred Gillett, ed. L. Caroff, L. J. Moon, D. E. Backman, \& E. Praton (San Francisco: ASP), 9

Backman, D. E., \& Paresce, F. 1993, in Protostars and Planets III, ed. E. Levy \& J. I. Lunine (Tucson: Univ. Arizona Press), 1253

Baraffe, I., Chabrier, G., Allard, F., \& Hauschildt, P. H. 1998, A\&A, 337, 403

Beichman, C. A., et al. 2005a, ApJ, 622, 1160

. 2005b, ApJ, 626, 1061

Bryden, G., et al. 2006, ApJ, 636, 1098

Burns, J. A., Lamy, P. L., \& Soter, S. 1979, Icarus, 40, 1

Carpenter, J. M., Wolf, S., Schreyer, K., Launhardt, R., \& Henning, T. 2005, AJ, 129, 1049

Chen, C. H., \& Jura, M. 2001, ApJ, 560, L171

Chen, C. H., Jura, M., Gordon, K. D., \& Blaylock, M. 2005, ApJ, 623, 493

Cohen, M., Megeath, S. T., Hammersley, P. L., Martín-Luis, F., \& Stauffer, J. 2003, AJ, 125, 2645

Crovisier, J., Leech, K., Bockelee-Morvan, D., Brooke, T. Y., Hanner, M. S., Altieri, B., Keller, H. U., \& Lellouch, E. 1997, Science, 275, 1904

Crovisier, J., et al. 1996, A\&A, 315, L385

D’Antona, F., \& Mazzitelli, I. 1997, Mem. Soc. Astron. Italiana, 68, 807

Decin, G., Dominik, C., Malfait, K., Mayor, M., \& Waelkens, C. 2000, A\&A, 357, 533

Decin, G., Dominik, C., Waters, L. B. F. M., \& Waelkens, C. 2003, ApJ, 598, 636

Dohnanyi, J. W. 1969, J. Geophys. Res, 74, 2531

Dominik, C., \& Decin, G. 2003, ApJ, 598, 626

Draine, B. T., \& Lee, H.-M. 1984, ApJ, 285, 89

Fazio, G., et al. 2004, ApJS, 154, 10

Fixsen, D. J., \& Dwek, E. 2002, ApJ, 578, 1009

Fraquelli, D. A., Schultz, A. B., Bushouse, H., Hart, H. M., \& Vener, P. 2004, PASP, 116, 55

Gordon, K. D., et al. 2004, in Proc. SPIE, 5487, 177

2005, PASP, 117, 503

Gorlova, N., et al. 2004, ApJS, 154, 448

Grogan, K., Dermott, S. F., \& Durda, D. D. 2001, Icarus, 152, 251

Gustafson, B. A. S. 1994, Ann. Rev. Earth Planet. Sci., 22, 553

Habing, H. J., et al. 2001, A\&A, 365, 545

Hines, D. C., Low, F. J., Schneider, G., Chandler, C. J., Plait, P., \& Smith, P. S. 2004a, in ASP Conf. Ser. 324, Debris Disks and the Formation of Planets: A Symposium in Memory of Fred Gillet, ed. L. Caroff, L. J. Moon, D. E. Backman, \& E. Praton (San Francisco: ASP), 262

Hines, D. C., et al. 2004b, FEPS Explanatory Suppl. V1.1 (Pasadena: SSC) . 2005, FEPS Explanatory Suppl. V2.1 (Pasadena: SSC)

Higdon, S. J. U., et al. 2004, PASP, 116, 975

Hollenbach, D., et al. 2005, ApJ, 631, 1180

Houck, J., et al. 2004, ApJS, 154, 18

Houk, N., \& Smith-Moore, M. 1988, Michigan Catalogue of Two-dimensional Spectral Types for the HD Stars, Vol. 4 (Ann Arbor: Univ. Michigan)

Jura, M., Malkan, M., White, R., Telesco, C., Pina, R., \& Fisher, R. S. 1998, ApJ, 505, 897

Jura, M., Zuckerman, B., Becklin, E. E., \& Smith, R. C. 1993, ApJ, 418, L37

Kenyon, S. J., \& Bromley, B. C. 2004, ApJ, 602, L133 2005, AJ, 130, 269

Kim, J. S., et al. 2005, ApJ, 632, 659

Klahr, H. H., \& Lin, D. N. C. 2001, ApJ, 554, 1095

Klein, T., Münker, C., Mezger, K., \& Palme, H. 2002, Nature, 418, 952

Koerner, D. W., Jensen, E. L. N., Cruz, K. L., Guild, T. B., \& Gultekin, K. 2000, ApJ, 533, L37

Lagrange, A.-M., Backman, D. E., \& Artymowicz, P. 2000, Protostars and Planets IV, ed. V. Mannings, A. Boss, \& S. Russell (Tucson: Univ. Arizona Press), 639

Laor, A., \& Draine, B. T. 1993, ApJ, 402, 441

Laureijs, R. J., Jourdain de Muizon, M., Leech, K., Siebenmorgen, R., Dominik, C., Habing, H. J., Trams, N., \& Kessler, M. F. 2002, A\&A, 387, 285 Low, F. J., Hines, D. C., \& Schneider, G. 1999, ApJ, 520, L45

Low, F. J., Smith, P. S., Werner, M., Chen, C., Krause, V., Jura, M., \& Hines, D. C. 2005, ApJ, 631, 1170
Markwardt, C. B. 2003, IDL Curve Fitting and Function Optimization, http:// cow.physics.wisc.edu/ craigm/idl/fitting.html

Marquardt, D. W. 1963, J. Soc. Ind. Appl. Math., 11, 431

Meyer, M. R., Backman, D., Mamajek, E. E., Herrera, V. M., Hinz, P., Carpenter, J. M., Hoffman, W., \& Hora, J. 2001, BAAS, 33, 1420

Meyer, M. R., et al. 2004, ApJS, 154, 422

Moro-Martín, A., \& Malhotra, R. 2002, AJ, 124, 2305 . 2003, AJ, 125, 2255

Moro-Martín, A., Wolf, S., \& Malhotra, R. 2005, ApJ, 621, 1079

Nesvorný, D., Bottke, W. F., Dones, L., \& Levison, H. F. 2002, Nature, 417, 720

Nesvorný, D., Bottke, W. F., Levison, H. F., \& Dones, L. 2003, ApJ, 591, 486

Nordström, B., et al. 2004, A\&A, 418, 989

Perryman, M. A. C., \& ESA. 1997, The Hipparcos and Tycho Catalogues (ESA SP-1200; Noordwijk: ESA)

Quijada, M. A., Marx, C. T., Arendt, R. G., \& Moseley, S. H. 2004, Proc. SPIE, 5487,244

Reach, W. T. 1992, ApJ, 392, 289

Reach, W. T., Morris, P., Boulanger, F., \& Okumura, K. 2003, Icarus, 164, 384

Reach, W. T., et al. 2005, PASP, 117, 978

Rieke, G. H., et al. 2004, ApJS, 154, 25

Schneider, G., et al. 1999, ApJ, 513, L127

Schneider, G., \& Stobie, E. 2002, in ASP Conf. Ser. 281, ADASS XI, ed. D. A.

Bohlender, D. Durand, \& T. H. Handley (San Francisco: ASP), 382

Schütz, O., Meeus, G., \& Sterzik, M. F. 2005, A\&A, 431, 165

Silverstone, M. D., et al. 2006, ApJ, in press

Skinner, C. J., Barlow, M. J., \& Justtanont, K. 1992, MNRAS, 255, 31P

Sitko, M. L., Lynch, D. K., \& Russell, R. W. 2000, AJ, 120, 2609

Soderblom, D. R., Henry, T. J., Shetrone, M. D., Jones, B. F., \& Saar, S. H. 1996, ApJ, 460, 984

Soderblom, D. R., et al. 1998, ApJ, 498, 385

Song, I., Zuckerman, B., \& Bessell, M. S. 2003, ApJ, 599, 342

Song, I., Zuckerman, B., Weinberger, A. J., \& Becklin, E. E. 2005, Nature, 436,363

Spangler, C., Sargent, A. I., Silverstone, M. D., Becklin, E. E., \& Zuckerman, B. 2001, ApJ, 555, 932

Stauffer, J. R., et al. 2005, AJ, 130, 1834

Stern, S. A. 1994, AJ, 108, 2312

Takeuchi, T., \& Artymowicz, P. 2001, ApJ, 557, 990

Tanaka, H., Inaba, S., \& Nakazawa, K. 1996, Icarus, 123, 450

Thébault, P., Augereau, J. C., \& Beust, H. 2003, A\&A, 408, 775

Torres, G., Stefanik, R. P., Latham, D. W., \& Mazeh, T. 1995, ApJ, 452, 870

Wahhaj, Z., Koerner, D. W., Backman, D. E., Werner, M. W., Serabyn, E., Ressler, M. E., \& Lis, D. C. 2005, ApJ, 618, 385

Walker, H. J., \& Wolstencroft, R. D. 1988, PASP, 100, 1509

Weinberger, A. J., Becklin, E. E., Zuckerman, B., \& Song, I. 2004, AJ, 127, 2246

Weingartner, J. C., \& Draine, B. T. 2001, ApJ, 548, 296

Werner, M. W., et al. 2004, ApJS, 154, 1

White, R. J., Gabor, J., \& Hillenbrand, L. A. 2006, ApJ, in press

Wichmann, R., Schmitt, J. H. M. M., \& Hubrig, S. 2003, A\&A, 399, 983

Williams, D. R., \& Wetherill, G. W. 1994, Icarus, 107, 117

Wilner, D. J., Holman, M. J., Kuchner, M. J., \& Ho, P. T. P. 2002, ApJ, 569, L115

Wolf, S., \& Hillenbrand, L. A. 2003, ApJ, 596, 603

Wooden, D. H., Harker, D. E., Woodward, C. E., Butner, H. M., Koike, C., Witteborn, F. C., \& McMurtry, C. W. 1999a, ApJ, 517, 1034

Wooden, D. H., Harker, D. E., Woodward, C. E., Koike, C., \& Butner, H. M. 1999b, Earth Moon Planets, 78, 285

Wyatt, M. C. 2005, A\&A, 433, 1007

Wyatt, M. C., \& Dent, W. R. F. 2002, MNRAS, 334, 589

Wyatt, M. C., Dermott, S. F., Telesco, C. M., Fisher, R. S., Grogan, K., Holmes, E. K., \& Piña, R. K. 1999, ApJ, 527, 918

Yin, Q., et al. 2002, Nature, 418, 949

Zacharias, N., Urban, S. E., Zacharias, M. I., Wycoff, G. L., Hall, D. M., Monet, D. G., \& Rafferty, T. J. 2004, AJ, 127, 3043

Zhang, B., \& Sigurdsson, S. 2003, ApJ, 596, L95

Zuckerman, B. 2001, ARA\&A, 39, 549

Zuckerman, B., \& Becklin, E. E. 1993, ApJ, 406, L25

Zuckerman, B., \& Webb, R. A. 2000, ApJ, 535, 959

Note added in proof.- While corpuscular drag forces are thought to be small for the present-day Sun $\left(F_{\text {wind }} / F_{\mathrm{P}-\mathrm{R}} \sim 0.35\right.$; Gustafson 1994), the effect might be much larger in younger systems with larger X-ray flux such as HD 12039 (B. E. Wood, H.-R. Müller, G. P. Zank, \& J. L. Linsky, ApJ, 574, 412 [2002]). We thank M. Jura for pointing out the potential importance of corpuscular drag at young ages, which was minimized in the original manuscript. 